\title{
Improving Transplant Opportunities for Patients who are Sensitized (ITOPS): Protocol for a Feasibility, Randomized, Controlled, Phase III Clinical Trial
}

\author{
Sian Griffin ( $\nabla$ sian.griffin2@wales.nhs.uk) \\ University Hospital of Wales https://orcid.org/0000-0001-5860-9036 \\ Tracey Rees \\ Welsh Transplantation and Immunogenetics Laboratory \\ Samaher Sweity \\ NHSBT Clinical Trials Unit \\ Helen Williams \\ University Hospital of Wales \\ Laura Pankhurst \\ NHSBT Clinical Trials Unit \\ Brendan Clarke \\ St James's Hospital, Leeds \\ Dave Collett \\ NHSBT Clinical Trials Unit \\ Duha llyas \\ St James's Hospital, Leeds \\ Maureen Edgar \\ University Hospital of Wales \\ Claire Dyer \\ NHSBT Clinical Trials Unit \\ Sunil Daga \\ St James's Hospital, Leeds \\ Rommel Ravanan \\ Southmead Hospital, Bristol
}

\section{Study Protocol}

Keywords: Kidney transplant, HLA antibodies, Highly Sensitised, Rituximab, Bortezomib, Plasmapheresis

Posted Date: December 12th, 2020

DOl: https://doi.org/10.21203/rs.3.rs-122212/v1

License: (c) (i) This work is licensed under a Creative Commons Attribution 4.0 International License. Read Full License 


\section{Abstract}

- Background: Kidney transplantation offers a better quality of life and a clear survival advantage compared to long-term dialysis. However, rates of transplantation are low for highly sensitized patients (HSP) who have a broad specificity of human leucocyte antigen (HLA) specific antibodies. This is because the presence of pre-formed HLA Donor Specific Antibodies can result in hyperacute rejection and is an immunological veto to transplantation. The proportion of eligible donors therefore decreases with increasing sensitization, resulting in increased waiting time prior to transplantation. This study aims to assess the efficacy of a combination of B cell depletion and proteasome inhibition, together with antibody removal and steroids, to reduce HLA antibodies in HSP, predicted to improve rates of transplantation, and to determine the feasibility of conducting a future definitive trial.

- Methods: ITOPS is a multi-center, randomized parallel, non-blinded, controlled phase III feasibility trial. The participants will be allocated in a 1:1 ratio to either the trial intervention (rituximab followed by 1-2 cycles of plasmapheresis, bortezomib and dexamethasone) or control group (no intervention).

- Discussion: The primary outcome is the proportion of patients achieving an absolute reduction in sensitization (calculated reaction frequency, cRF) of at least $10 \%$ at 12 weeks following their last intervention, compared to the control group.

Secondary outcomes include recruitment and transplantation rates, durability of change and absolute percentage change in CRF, and acceptability of the intervention. We will proceed to development of a larger trial if at least $50 \%$ of the participants in the intervention arm achieve an absolute reduction in CRF of a minimum of $10 \%$ at 12 weeks following their last intervention. If the proportion is $<50 \%$ in the intervention arm but $50 \%$ is included within the $95 \%$ confidence interval, then consideration to proceed to a larger randomized trial will be given alongside other aspects of feasibility.

- Trial Registration: International Standard Randomized Controlled Trial Number ISRCTN66441193

https://doi.org/10.1186/ISRCTN66441193

\section{Introduction}

\section{Background and rationale $\{6 a\}$}

Despite the advantages of kidney transplantation compared to long term dialysis, rates of transplantation are low for sensitized patients with high levels and breadth of human leucocyte antigen (HLA) specific antibodies(1). The production of these antibodies is stimulated by exposure or sensitization to non-self HLA, and frequently occurs following pregnancy, blood transfusion and previous transplantation (2). In terms of their risk to the graft, pre-formed HLA Donor Specific Antibodies are heterogeneous (3-8). The Luminex Single Antigen Bead assay allows semi-quantification of antibody concentration (expressed as Mean Fluorescence Intensity, MFI) and serial monitoring of antibodies of individual HLA specificities. An MFI $<2000$ is generally associated with a negative flow cytometric cross match and is a safe threshold for transplantation.

Highly Sensitized (HS) patients (those with a calculated Reaction Frequency (cRF) $\geq 85 \%$ ) are difficult to match to a compatible donor. Compared to recipients with a lower degree of sensitization, HS patients tend to be younger, are more likely to be female and their waiting time before receiving an offer of a deceased donor transplant is longer (Table 1). 
Table 1

Sensitized patients on the UK renal transplant waiting list, 31/12/16.

\begin{tabular}{|c|c|c|c|c|}
\hline & Number of patients & $\begin{array}{l}\text { Median Age } \\
\text { (years) }\end{array}$ & $\begin{array}{l}\text { Female } \\
(\%)\end{array}$ & Median waiting time to transplant* (days) \\
\hline $\mathrm{cRF}<85 \%$ & 3851 & 54 & 35 & 843 \\
\hline \multicolumn{5}{|l|}{ - $74 \%$ of waiting list } \\
\hline $85 \leq \mathrm{cRF}<94 \%$ & 289 & 51 & 67 & 1331 \\
\hline \multicolumn{5}{|l|}{ - $6 \%$ of waiting list } \\
\hline$c R F \geq 95 \%$ & 1049 & 49 & 56 & 2074 \\
\hline - $20 \%$ of waiting list & & & & \\
\hline
\end{tabular}

To define those participants most likely to benefit from a reduction in CRF (in terms of subsequent likelihood of receiving the offer of a transplant), we data from the UK Transplant Registry (UKTR), held National Health Service Blood and Transplant (NHSBT), to analyze transplants performed in the United Kingdom (UK) between 01/01/2012 and 31/12/2015 by waiting time and sensitization (Table 2). This demonstrates that for those patients who have already accrued at least 3 years waiting time, a relatively modest decrease in cRF can substantially increase the chance of being offered a transplant.

Table 2

Chance of a deceased donor transplant in the following year, stratified by waiting time and sensitization, including patients in the waiting list between 01/01/2012 and 31/12/2015.

\begin{tabular}{|c|c|c|}
\hline \multicolumn{3}{|c|}{ Deceased Donor } \\
\hline CRF & Waiting time & Chance of a transplant in the next year \\
\hline$\geq 95 \%$ & \multirow[t]{4}{*}{3 years } & $9 \%$ \\
\hline $90-94 \%$ & & $9 \%$ \\
\hline $85-89 \%$ & & $11 \%$ \\
\hline $80-84 \%$ & & $21 \%$ \\
\hline$\geq 95 \%$ & \multirow[t]{3}{*}{5 years } & $9 \%$ \\
\hline $90-94 \%$ & & $19 \%$ \\
\hline $85-89 \%$ & & $26 \%$ \\
\hline
\end{tabular}

For those with a living donor, the UK Living Kidney Sharing Scheme (UKLKSS) offers the chance of receiving a compatible transplant. However, for those with a cRF $\geq 95 \%$ the chance of being offered a match through the UKLKSS is relatively small. We analyzed the likelihood of a match (ever) in the UKLKSS based on all patients included between 2012-2015, which also confirmed the impact of reduction in cRF (Table 3). 
Table 3

Chance of a match in the UK Living

Kidney Sharing Scheme by

sensitization.

\begin{tabular}{|ll|}
\hline \multicolumn{2}{|l|}{ UK Living Kidney Sharing Scheme } \\
\hline CRF & Chance of a transplant \\
\hline $100 \%$ & $4 \%$ \\
\hline $95-99 \%$ & $21 \%$ \\
\hline $85-94 \%$ & $42 \%$ \\
\hline $10-84 \%$ & $47 \%$ \\
\hline $0-9 \%$ & $37 \%$ \\
\hline
\end{tabular}

In addition to the personal, social and economic impact, long term dialysis is associated with an accumulation of related morbidity and mortality (9). UKTR data from 2018-19 suggests $10 \%$ of HS patients will die within five years of joining the transplant waiting list, and a further $12 \%$ will be removed from the transplant waiting list due to deteriorating health, so that transplantation is no longer an option (Annual activity report 2018-19, NHSBT, UK). This has provided the impetus for the development of protocols for modification of HLA antibody profile prior to transplantation, referred to as desensitization.

Desensitization strategies have used combinations of antibody removal by plasma exchange or immunoadsorption, antibody modulation by intravenous immunoglobulin (IVIg), depletion of B cells by CD20 blockade (by rituximab) and targeting plasma cells by proteasome inhibition (bortezomib and more recently carfilzomib) (10-14) (15-20). The CedarsSinai group has the greatest experience of transplanting patients following desensitization with protocols using Rituximab and IVIg $(11,13,14,21)$. However, the suppression of HLA antibodies using this approach is relatively short lived, and rates of antibody mediated rejection following transplantation are relatively high (10,22-25). As might be expected, treatment with Rituximab and IVIg has no effect on splenic memory B cells and plasma cells (26). Bortezomib has been used both to treat antibody mediated rejection (27-30), and as part of a desensitization regimen to lower HLA antibody levels prior to transplantation $(15,17-19,27,31,32)$.

The published case reports and trials that have used a combination desensitization protocol with both rituximab and bortezomib have shown the greatest reduction in HLA antibodies and increase in rates of transplantation with good posttransplant outcomes $(17-19,32)$. The success of these trials has informed the design of this trial, with the aim of optimizing response rates without unacceptable toxicity. The overall aim of our study is hence to test efficacy and durability of reduction in antibodies using a combination of desensitization treatments in a prospective controlled approach against the current standard of care (SOC).

\section{Objectives $\{7\}$}

The primary objective is to assess the efficacy of a combination of B cell depletion and proteasome inhibition, together with antibody removal and steroids, to reduce HLA antibodies in HS patients.

The secondary objectives are:

1. To confirm that the expected number of patients can be recruited in the time frame of the trial.

2. To confirm that the intervention is sufficient to result in a durable fall in $\mathrm{cRF}$.

3. To confirm that B cell depletion persists at 12 weeks following intervention.

4. To determine whether the intervention impacts on Quality of Life.

5. To confirm whether any transplants arise as a consequence of the intervention. 
6. To confirm incidence of peripheral neuropathy (any grade).

\section{Trial Design \{8\}}

The ITOPS design is a parallel two arm feasibility trial, with participants being allocated in a 1:1 ratio to the trial intervention versus control group. The is no blinding in the study.

\section{Methods: Participants, Interventions And Outcomes Study setting $\{9\}$}

The study will be conducted in the renal departments of three UK transplant centres: University Hospital of Wales, Cardiff (Cardiff and Vale University Health Board); St James's Hospital, Leeds (Leeds Teaching Hospital NHS Trust) and Southmead Hospital, Bristol (North Bristol NHS Trust).

\section{Eligibility criteria $\{10\}$}

Highly sensitized patients who have completed the standard medical and surgical assessments prior to being considered eligible for kidney transplantation will be included according to criteria detailed below.

\section{Inclusion criteria:}

1. Male or female, aged 18 years or older.

2. Able to give informed consent and willing to fulfil trial requirements.

3. Have completed the necessary assessments to receive a renal transplant.

4. In stable health as determined by the Investigator based on medical history and laboratory tests during the screening period.

5. Negative serological test for hepatitis B (surface antigen and core antibody) and hepatitis $C$ within the last 6 months, and Human Immunodeficiency Virus (HIV) within the last 12 months.

6. Documented immune status to varicella zoster virus (VZV) (at any time).

7. Female participant of childbearing potential must be willing to use a highly effective method of contraception for one year following rituximab (this period will also include the recommended three-month period for avoidance of pregnancy following bortezomib). Male participants must be willing to use a highly effective form of contraception for 3 months post last dose of bortezomib.

8. Persistent (for at least one year) and stable (tested on at least three occasions over the preceding 18 months) circulating HLA antibodies defined by Luminex Single Antigen Bead analysis at the time of recruitment.

9. Recipients awaiting deceased donor transplantation:

- $\mathrm{CRF} \geq 85 \%$ and at least 3 years on the transplant waiting list.

Recipients with an HLA incompatible living donor who have been considered for enrolment in the UK Living Kidney Sharing Scheme:

- $\mathrm{cRF}$ is $\geq 95 \%$ or,

- $\mathrm{CRF}$ is $\geq 85 \%$ and they have received no offers after three runs in the UKLKSS.

\section{Exclusion criteria:}

1. Pregnancy, planned pregnancy during the trial period, or current breast feeding. 
2. Active viral, bacterial or fungal infection precluding immunosuppression.

3. Active malignant disease.

4. Patients listed for transplantation of any organ other than kidney alone.

5. History of, or active, clinically significant, respiratory, gastrointestinal (including pancreatitis), hepatic, cardiac, neurological, psychiatric, musculoskeletal, genitourinary, dermatological, or other disorder that, in the Investigator's opinion, could affect the conduct of the trial.

6. History of non-adherence, alcohol or substance abuse that, in the judgment of the Investigator, may impair or risk the subject's full participation in the trial.

7. Positive serological test for hepatitis B, C or HIV.

8. Negative serological test for VZV.

9. Previous graft loss to recurrent primary disease within 2 years of transplantation.

10. Documented intolerance of bortezomib, rituximab or their excipients, or dexamethasone.

11. Persistent thrombocytopaenia, platelet count $<100 \times 10^{9} / \mathrm{L}$ for the past three consecutive months.

12. Persistent neutropaenia, absolute neutrophil count $<2 \times 10^{9} / \mathrm{L}$ for the past three consecutive months.

13. Hypogammaglobulinaemia, serum immunoglobulin $\mathrm{G}(\mathrm{lgG})$ less than local laboratory lower limit of normal at screening.

14. Peripheral neuropathy of any grade (reported by symptoms or on clinical examination).

15. Currently involved in any other clinical trial of an investigational medicinal product (IMP) or have taken an IMP within 30 days prior to trial entry except the SIMPLIFIED trial (ISRCTN15087616 https://doi.org/10.1186/ISRCTN15087616. Natural vitamin D (cholecalciferol) versus standard care in patients receiving dialysis).

\section{Who will take informed consent? $\{26 \mathrm{a}\}$}

It is the responsibility of the Principle Investigator $(\mathrm{PI})$ at each site to give adequate oral and written information about the purposes and procedures of the study, information about data protection, the possible advantages and disadvantages of participation, and option to withdraw from the study at any time and without any given reason. Written informed consent must be obtained for all participants prior to any trial-related procedures. The right of the patient to refuse to participate without giving a reason will be respected.

All staff involved in trial-related activities will be authorized, trained and competent to do so according to the ethically approved protocol, principles of International Conference on Harmonization Good Clinical Practice (ICH GCP) and Declaration of Helsinki as detailed in the Medicines for Human Use (Clinical Trials) Regulations and associated amendments.

\section{Additional consent provisions for collection and use of participant data and biological specimens $\{26 \mathrm{~b}\}$}

Separate, specific, optional consent will be sought for collection of biological samples for exploratory analyses. A separate explanatory section is included in the Patient Information Sheet.

\section{Interventions}

\section{Explanation for the choice of comparators $\{6 \mathrm{~b}\}$}

There are no approved interventions for this group of patients, and the comparator is therefore current SOC, which is regular monitoring of HLA antibody levels. 


\section{Intervention arm:}

Day 0: Rituximab intravenously as a single dose of $1 \mathrm{~g}$. A reduced dose of $500 \mathrm{mg}$ will be administered to participants weighing less than $50 \mathrm{~kg}$.

Days 21, 24, 28, 31: Plasmapheresis (1.5 plasma volumes) followed by bortezomib $1.3 \mathrm{mg} / \mathrm{m} 2$ subcutaneously and dexamethasone $20 \mathrm{mg}$ orally.

If the fall in CRF is less than 10\% 12 weeks after the first treatment cycle of plasmapheresis, bortezomib and dexamethasone, a second cycle of treatment will be offered.

\section{Criteria for discontinuing or modifying allocated interventions $\{11 \mathrm{~b}\}$}

The dose of bortezomib may be modified based on the result of the full blood count (FBC) done prior to administration. A neutrophil count of $\geq 2 \times 10^{9} / \mathrm{L}$ and platelet count $\geq 100 \times 10^{9} / \mathrm{L}$ are prerequisites for inclusion in the trial. On subsequent days of treatment, if the neutrophil count is $\geq 0.5 \times 10^{9} / \mathrm{L}$ and platelet count $\geq 30 \times 10^{9} / \mathrm{L}$, that day's dose will be given. If the neutrophil count is $<0.5 \times 10^{9} / \mathrm{L}$ and/or the platelet count is $<30 \times 10^{9} / \mathrm{L}$, no further treatment will be given for that cycle. If a second cycle is considered, bortezomib will be given at half the original dose.

\section{Strategies to improve adherence to interventions $\{11 \mathrm{c}\}$}

The intervention is carried out in hospital under nursing supervision and hence non-adherence should be limited. Participants will receive prophylaxis against infection. The importance of this will be emphasized, together with ensuring supply of regular prescriptions. The trial and data managers will also monitor adherence through site visits and the data which is inputted onto the trial database. Any issues will be reported to and discussed at the trial management group meetings.

\section{Relevant concomitant care permitted or prohibited during the trial $\{11 d\}$}

Paracetamol (1 g, oral), chlorpheniramine (10 mg, intravenous) and hydrocortisone (100 mg, intravenous) are given prior to the infusion of rituximab.

Prophylaxis against infection is required for all patients in the intervention group as per local practice.

- Prophylaxis against pneumocystis jiroveci pneumonia for 6 months after rituximab:

First line agent - cotrimoxazole- dose as per local practice

- Prophylaxis against herpes simplex infection for three weeks beginning with the first dose of bortezomib for each cycle of the treatment:

First line agent - acyclovir, 200 mg daily

Regular prescriptions including anti-hypertensive drugs, phosphate binders, vitamin D and erythropoiesis stimulating agents should continue.

\section{Provisions for post-trial care $\{30\}$}

Once the trial is over, all participants will receive all standard care whilst on the waiting list and following transplantation. This is an NHS-sponsored research trial, and the NHS indemnity scheme therefore applies. If there is negligent harm during the clinical trial when the NHS body owes a duty of care to the person harmed, NHS indemnity covers NHS staff, medical academic staff with honorary contracts, and those conducting the trial. The NHS indemnity scheme does not cover nonnegligent harm. The liability of the manufacturers of rituximab and bortezomib is strictly limited to those claims arising from faulty manufacturing of the commercial product and not to any aspects of the conduct of the trial. 


\section{Outcomes $\{12\}$}

\section{Primary Outcome}

The proportion of patients achieving an absolute reduction in CRF of at least $10 \%$ at 12 weeks following their last intervention, compared to control group who have received no intervention.

For the purposes of calculating reaction frequency in the ITOPS trial, all HLA A, B and DR antibodies with an MFI $\geq 2000$ when tested in the local H\&l laboratory will be classified as positive. HLA CW, DP and DQ antibodies will be classified as positive according to local practice. HLA A, B and DR antibodies with an MFI<2000 at 12 weeks (and other time-points for the purpose of other relevant secondary outcomes) will be removed from unacceptable antigen listing.

If the MFI of an HLA A, B or DR antibody rises above an MFI of 2500 following previous de-listing, they will be listed again. A cut off of 2500 is used for re-listing as there is greater variability in antibody level at these relatively low concentrations.

\section{Secondary Outcomes:}

1. The proportion of eligible participants who consent to the trial.

2. Confirmation that at 12 weeks post-final intervention there has been an absolute fall in cRF of least $10 \%$ in at least $50 \%$ of the participants treated, compared to CRF at enrolment.

3. The proportion of patients in the intervention and control groups achieving an absolute reduction in $\mathrm{cRF}$ of at least $10 \%$ at 24 weeks following their last intervention or on routine monitoring, as compared to CRF at enrolment.

4. The proportion of patients in the intervention and control groups achieving an absolute reduction in cRF of at least $10 \%$ at 48 weeks following their last intervention or on routine monitoring, as compared to cRF at enrolment.

5. Compared to cRF at enrolment, the percentage decrease in CRF at 12, 24, 36 and 48 weeks following final treatment in intervention group or on routine monitoring.

6. The percentage of participants in the intervention group with B cell depletion at 12 weeks after the first cycle of B cell depletion is defined as an absolute reduction in $B$ cell count to equal to or less than $5 \%$ the $B$ cell count at enrolment for that individual participant.

7. Patient acceptability - quality of life questionnaires (European Quality of Life - 5 dimensions, EQ-5D and Kidney Disease Quality of Life - Short Form, KDQoL-will be completed at recruitment and 18 months after enrolment for both intervention and control groups (or 90 days after transplantation, whichever is either face to face or by post.

8. The number of participants receiving a transplant during the study period from either a deceased or a living donor (with confirmation that any increase in offer rate was as a result of change in CRF as per ITOPS protocol).

9. The number of participants developing any grade neuropathy following each cycle of treatment.

\section{Participant timeline $\{13\}$}

Figure 1. Participant timeline.

\section{Sample size $\{14\}$}

As this is a small-scale feasibility study, a formal sample size calculation was not performed. Based on the expected pool of potentially eligible participants (64 patients when the protocol was written: 15 at the University Hospital of Wales in Cardiff, 23 at Southmead Hospital in Bristol and 26 at St James' Hospital in Leeds), expected rates of potential participants who are confirmed to be ineligible (20\%), a sample size of 38 participants was proposed to be recruited over a 12 months period.

This will allow the trial to determine whether $50 \%$ of participants in the intervention group achieve an absolute reduction in CRF of at least $10 \%$ after 12 weeks of their last intervention as compared to cRF at enrolment, which would be a clinically 
important outcome. Specifically, taking $n=19$, an exact $95 \%$ confidence interval for the true proportion of participants who achieve an absolute reduction in cRF of at least $10 \%$ in intervention arm is [27\%, $73 \%]$.

\section{Recruitment $\{15\}$}

All potentially eligible participants will be identified by pre-screening the inclusion and exclusion criteria against the active transplant waiting list at each centre. The initial approach will be by a member of the clinical team responsible for the patients' care, followed by a more detailed discussion with the trial team. The intervention will be scheduled according to the participants convenience.

\section{Assignment Of Interventions: Allocation Sequence generation $\{16 a\}$}

The allocation sequence will be produced by the trial statistician using SAS statistical software. The allocation sequence will have a varying undisclosed block size and will be stratified by site. Participants will be allocated to a 1:1 ratio to the trial intervention (rituximab, plasmapheresis, bortezomib and dexamethasone) versus control (standard care) group.

\section{Concealment mechanism \{16b\}}

Participants will be randomized using Sealed Envelope (www.sealedenvelope.com), which is an interactive web-based randomization service, and the allocation sequence will be uploaded into it by the trial statistician. Allocation concealment will be ensured as the list will be held securely by the statistician and within the randomization system only. The participant's screening log number and initials will need to be provided to Sealed Envelope to obtain randomization code and treatment group. The trial manager will be notified of new randomizations by Sealed Envelope.

\section{Implementation \{16c\}}

Participant allocation will be made using Sealed Envelope, which will allocate the next randomization code and treatment group in sequence at each center. The PI at each site will enroll the patient into the trial. Randomization may be done by any member of the research team.

\section{Assignment Of Interventions: Blinding Who will be blinded $\{17 \mathrm{a}\}$}

No-one within the trial is blinded to the intervention.

Procedure for unblinding if needed $\{17 b\}$

Not applicable.

\section{Data Collection And Management \\ Plans for assessment and collection of outcomes \{18a\}}

Plans to promote participant retention and complete follow-up $\{18 b\}$

As trial participants will be attending for regular dialysis retention of participants in the trial is expected to be high.

Data management $\{19\}$ 
Individual patient data for the ITOPS trial will be captured on an electronic Case Report Form (eCRF). Only the data required by the protocol will be captured in the eCRF. Each PI and trial site will keep a record of all participating patients with sufficient information to link records, including eCRFs, hospital notes and samples, and all original signed informed consent forms.

The PI has overall responsibility for data collection at their site. Participant data will be entered electronically onto the trial database designed and administered by the NHSBT CTU data management team using MACRO-v4.4.0.6647, a commercially available FDA 21 CRF Part 11 compliant clinical trial database system produced by Elsevier. The MACRO database will have in-built validation rules and conditions, to help optimize the quality of the data. In addition, the designated H\&I scientist at each site will be responsible for completion of a standardized and anonymized spreadsheet detailing the MFI of all HLA antibodies at each time point, which will be sent electronically (using password protected spreadsheet) to the NHSBT CTU at the end of the study for analysis.

The NHSBT CTU staff will be in regular contact with local site personnel to check on progress and to help with any queries that may arise. Incoming electronic forms will be checked for completeness, consistency, timelines, and compliance with the protocol.

\section{Confidentiality $\{27\}$}

All investigators and trial site staff must comply with the requirements of the UK Data Protection regulations with regards to the collection, storage, processing, and disclosure of personal information and will uphold the regulations' core principles. The confidentiality standards will be maintained by coding each patient enrolled in the trial through assignment of a unique patient identification number.

\section{Plans for collection, laboratory evaluation and storage of biological specimens for genetic or molecular analysis in this trial/future use $\{33\}$}

Several recent studies have demonstrated that the cytokine profile, lymphocyte immunophenotype or detailed antibody characteristics of an individual may be predictive of response to desensitization and subsequent outcome (32-37). There will therefore be a parallel collection of clinical samples for mechanistic studies, at time points from randomization until one year following the final intervention. These will include blood samples and the plasmapheresis effluent obtained from the first exchange of each cycle. A separate funding application will be made for the assays to be performed. Any results from the parallel studies will be correlated with clinical outcomes, with the aim of increasing understanding of the response of HLA sensitized patients to proteasome inhibition, enabling future risk stratification and prediction of those more likely to respond to this intervention.

These samples will be stored locally for the duration of the study, before shipping to Cardiff, or the center performing the specific assays of interest. In the longer term, any remaining samples will be shipped and stored in the Wales Kidney Research Tissue Bank in the Institute of Nephrology, University Hospital of Wales, Cardiff University, Cardiff. The necessary HTA approvals are in place for sample storage.

Analyses of these will form a separate future study and will not influence decision making in the current trial. Participation in this sub-trial is optional and specific consent will be sought. Participants who decline will remain eligible for inclusion in the ITOPS trial.

\section{Statistical Methods}

\section{Statistical methods for primary and secondary outcomes $\{20 \mathrm{a}\}$}


Full details of the statistical analyses will be specified in the Statistical Analysis Plan. This section summarizes the main points.

The analysis will be performed on intention to treat basis and will include all randomized participants on whom values of a response variable have been obtained. The data will be analyzed according to the treatment arm to which the participant was randomized.

Participants who did not meet all the inclusion criteria and/or met at least one of the exclusion criteria will be considered as randomized in error. Participants who were randomized to the intervention group but did not receive any trial treatment and participants who were randomized to the control group but received the trial treatment will be considered as protocol deviations. Participants randomized in error and participants where the protocol was not followed as specified will also be considered as protocol deviations. Participants randomized in error, participants with protocol deviation, and those withdrawn will be included in intention to treat analysis.

As this is a small-scale feasibility trial and all centers will follow the same protocol, no adjustment will be made for any center differences. Equally this trial has not been powered to detect differences between arms of the trial. Hence no statistical testing will be performed.

\section{Analysis of Primary and Secondary outcomes}

The proportion (and exact Binomial 95\% confidence interval) of participants achieving an absolute reduction in cRF of at least $10 \%$ at 12 weeks following their last intervention or on routine monitoring, as compared to cRF at enrolment. This will be calculated for each trial arm and overall. We will consider this study feasible and worthy of further development into a larger randomized trial if the point estimate for achieving a $10 \%$ reduction is $\geq 50 \%$ in the intervention arm. If the estimate is $<50 \%$ in the intervention arm but $50 \%$ is included within the $95 \%$ confidence interval, then consideration to proceed to a larger randomized trial will be given alongside other aspects of feasibility.

Recruitment and dropout rates will be presented by center. Serious infectious episodes and serious adverse events will be summarized. The number of participants in the intervention group developing any grade neuropathy following each cycle of treatment will be reported.

The method used for the analysis of the proportion of participants achieving an absolute reduction in cRF of at least $10 \%$ at 24 weeks and at 48 weeks following their last intervention or on routine monitoring, as compared to cRF at enrolment, will be similar to that described for the primary outcome. As compared to CRF at enrolment, mean change in cRF at 12, 24, 36and 48 weeks following the last treatment in intervention group or on routine monitoring in control group will be presented with the standard deviation. The difference in mean change between arms will be presented for 12, 24, 36 and 48 weeks separately, along with a $95 \%$ confidence interval.

The number, proportion and exact Binomial 95\% confidence interval will be presented for participants receiving transplant and for those participants in the intervention group with B cell depletion at 12 weeks after completing the first cycle of treatment. Quality of Life will be assessed using EQ-5D-5L and KDQoL-SF questionnaires. The proportional change from enrolment in Quality of Life at 18 months following enrolment (or 90 days after transplantation, whichever is earlier) will be calculated for each participant and from which the median and interquartile range will be presented.

\section{Other analyses}

The analysis with MFI cut off point of 2000 will be the primary analysis. However, this is a relatively stringent cut-off, and if any change in $\mathrm{CRF}$ is relatively modest the analysis will be rerun using an MFI cut off value of 3000 . This data will be available as part of the luminex SAB measurement performed throughout the trial. This secondary analysis will be for exploratory purposes only and it will not influence the conclusions of the trial.

Page $11 / 24$ 


\section{Interim analyses $\{21 \mathrm{~b}\}$}

No interim analyses are planned.

\section{Methods for additional analyses (e.g. subgroup analyses) \{20b\}}

No subgroup analyses are planned.

Methods in analysis to handle protocol non-adherence and any statistical methods to handle missing data $\{20 \mathrm{c}\}$

Please see 20a and further details to be specified in the statistical analysis plan. All missing data will be assumed to be missing at random and missing data will not be imputed.

\section{Plans to give access to the full protocol, participant level-data and statistical code $\{31 \mathrm{c}\}$}

The Trial Steering Committee (TSC), which has representation from all participating sites, will have access to the final trial dataset. Custody of the final data set will reside with the $\mathrm{Cl}$ and NHSBT CTU (for audit purposes). Access to the final data set for additional analyses will be permitted under the agreement of the TSC, and according to the trial publication policy.

\section{Oversight And Monitoring}

\section{Composition of the coordinating centre and trial steering committee $5 d$}

The Trial Management Group (TMG) has been set up by the $\mathrm{Cl}$ and will include the lead investigators from the other participating sites (Bristol and Leeds), members of the NHSBT CTU (trial manager, operations manager, data manager and statistician) and the Sponsor representative. The TMG convenes regularly, either face-to-face or by teleconference to discuss recruitment and other practical aspects of the trial. The day-to-day management of the trial is coordinated through the NHSBT CTU and the $\mathrm{Cl}$.

A TSC with an independent Chair, statistician, clinician, and patient representatives and will be responsible for overseeing the progress of the trial. The TSC will convene biannually either in person or by teleconference. The TSC will focus on progress of the trial, patient safety, and the consideration of new information relevant to the research question. The TSC will provide advice, through its Chair, to the $\mathrm{Cl}$, the Trial Sponsor, the Trial funder, and the Host Institution. The ultimate decision on continuation of the trial lies with the TSC. The TSC Charter includes details of membership as well as roles and responsibilities of the committees' members.

\section{Composition of the data monitoring committee, its role and reporting structure $\{21 \mathrm{a}\}$}

The NHSBT CTU has a core Independent Data Monitoring Committee (DMC) for its transplantation-related trials. The group will act as DMC to this trial, with the primary role of overseeing safety int the trial. They will periodically review trial data by arm and overall, to determine patterns and trend of events, or to identify safety issues. They will provide recommendations to the Chair of the TSC on whether the trial should continue and can recommend premature closure of the trial. The DMC Charter includes details of membership as well as roles and responsibilities of the committees' members.

Table 4. Schedule of study events

Table 4a. Treatment period schedule 1 


\begin{tabular}{|c|c|c|c|c|c|c|c|}
\hline \multirow[t]{2}{*}{ TIMEPOINT } & \multirow[t]{2}{*}{$\begin{array}{l}\text { Pre- } \\
\text { screening }\end{array}$} & \multirow{2}{*}{$\begin{array}{l}\text { Baseline }^{1} \\
\text { V1 }\end{array}$} & \multicolumn{5}{|c|}{$\begin{array}{l}\text { Treatment ( } D=\text { day, Wk=Week, } \\
\text { V=visit) }\end{array}$} \\
\hline & & & $\mathrm{V} 2^{1}$ & $\mathrm{~V}^{2}{ }^{2}$ & V4 & V5 & V6 \\
\hline & & & DO & $\mathrm{D} 21^{3}$ & $\mathrm{D} 24^{4}$ & $\mathrm{D} 28^{4}$ & D31 ${ }^{4}$ \\
\hline $\begin{array}{l}\text { Confirmation of Sensitization and Waiting Time on } \\
\text { Transplant List }\end{array}$ & $\mathrm{X}$ & & & & & & \\
\hline Informed Consent ${ }^{5}$ & & $\mathrm{x}$ & & & & & \\
\hline $\begin{array}{l}\text { History and physical examination/ Concomitant } \\
\text { medication }\end{array}$ & & $\mathbf{x}$ & $\mathrm{X}$ & $\mathrm{X}$ & $\mathrm{X}$ & $\mathbf{x}$ & $\mathrm{X}$ \\
\hline $\begin{array}{l}\text { Eligibility Screening with review of SOC tests: FBC, } \\
\text { clotting screen, bone profile, LFTs, magnesium, HBV } \\
\text { (HBsAg is SOC, HBcAb is not (and required before } \\
\text { treatment with rituximab), HCV, HIV, and VZV }\end{array}$ & $\mathbf{X}$ & $\mathbf{X}$ & & & & & \\
\hline Serum pregnancy test & & $x^{6}$ & & & & & \\
\hline $\begin{array}{l}\text { ECG, Total immunoglobulins (IgG) and B cell count } \\
\text { (for IG only) (all not SOC) }\end{array}$ & & $\mathrm{X}$ & & & & & \\
\hline EQ-5D ${ }^{7}$ and $K D Q o L-S F^{8}$ questionnaires & & $\mathrm{x}$ & & & & & \\
\hline FACT/GOG-Ntx $4^{9}$ questionnaire & & $\mathbf{x}$ & & & & & $X^{10}$ \\
\hline Randomization & & $\mathbf{x}$ & & & & & \\
\hline \multicolumn{8}{|l|}{ Interventions } \\
\hline Administration of Rituximab & & & $\mathrm{x}$ & & & & \\
\hline $\begin{array}{l}\text { Plasmapheresis followed by bortezomib with } \\
\text { dexamethasone }\end{array}$ & & & & $x$ & $\mathrm{X}$ & $x$ & $\mathrm{X}$ \\
\hline $\begin{array}{l}\text { FBC, clotting screen, bone profile (before each } \\
\text { treatment) }\end{array}$ & & & & $\mathrm{X}$ & $\mathrm{X}$ & $\mathrm{X}$ & $\mathbf{X}$ \\
\hline
\end{tabular}

1 - Visit 2 - If not within 4 weeks of visit 1, blood and pregnancy tests (if applicable) to be repeated. 2 - Insertion of vascath if required.

3 -This visit can be performed between 21 to 28 days post rituximab administration (i.e. day 0 ). Please adjust the subsequent visits accordingly if this visit has occurred between day 22-28 as per trial schedule.

4- Treatment given on days 24,28 and 31 can be given within +3 days if required, and same applies if a second cycle is needed.
6 - If V1 delayed beyond 4 weeks, pregnancy test to be repeated (if applicable).

7 - EQ-5D - European Quality of Life - 5 Dimensions.

8 - KDQOL-SF- Kidney Disease Quality of Life Short Form.

9 - FACT/GOG-Ntx4- Functional Assessment of Cancer

Therapy/Gynaecologic Oncology Group/Neurotoxicity.

10 - FACT/GOG-Ntx4 questionnaire to be completed at the end of cycle 1 (and end of cycle 2 if applicable) for the IG only.

5- Informed consent before any trial related activities. Non SOC tests can be done on V1 after consent. If required another visit/s can be scheduled to perform and/or review these.

Table 4b. Treatment period schedule 2 


\begin{tabular}{|c|c|c|c|c|c|c|c|c|c|c|c|c|c|}
\hline \multirow[t]{3}{*}{ TIMEPOINT } & \multirow[t]{3}{*}{$\begin{array}{l}\text { Pre- } \\
\text { screening }\end{array}$} & \multirow{3}{*}{$\begin{array}{l}\text { Baseline } \\
\text { V1 }\end{array}$} & \multicolumn{11}{|c|}{$\begin{array}{l}\text { Treatment } \\
(\mathrm{D}=\text { day, Wk=Week, } \mathrm{V}=\mathrm{visit})+/-3 \text { days for visits } 7-10 \text {, other subsequent } \\
\text { visits }+/ \text { - one } W k\end{array}$} \\
\hline & & & \multicolumn{5}{|c|}{ Treatment } & \multicolumn{6}{|c|}{ Post Treatment ${ }^{1}$} \\
\hline & & & V2 & V3 & V4 & V5 & V6 & V7 & v8 & V9 & V10 & V11 & V12 \\
\hline & & & DO & D21 & D24 & D28 & D31 & $\begin{array}{l}\text { Wk } \\
1\end{array}$ & $\begin{array}{l}\text { Wk } \\
2\end{array}$ & $\begin{array}{l}\text { Wk } \\
3\end{array}$ & $\begin{array}{l}\text { Wk } \\
4\end{array}$ & $\begin{array}{l}\text { Wk } \\
8\end{array}$ & $\begin{array}{l}\text { Wk } \\
12^{2}\end{array}$ \\
\hline $\begin{array}{l}\text { Concomitant } \\
\text { medication }\end{array}$ & & $x$ & $x$ & $x$ & $x$ & $x$ & $x$ & $x$ & $x$ & $x$ & $x$ & $x$ & $x$ \\
\hline $\begin{array}{l}\text { Total } \\
\text { immunoglobulins } \\
\text { (lgG) and B cell } \\
\text { count }\end{array}$ & & & & & & & & & & & & & $x^{3}$ \\
\hline \multicolumn{14}{|l|}{$\begin{array}{l}\text { Assessment of } \\
\text { HLA Antibodies } \\
\text { And cRF }\end{array}$} \\
\hline $\begin{array}{l}\text { Intervention } \\
\text { Group (IG) }\end{array}$ & $x^{4}$ & $x$ & & & & & & & $x$ & & $x$ & $x$ & $x$ \\
\hline $\begin{array}{l}\text { Control Group } \\
\text { (CG) }\end{array}$ & $x^{4}$ & $x$ & & & & & & & & & & & $x$ \\
\hline \multicolumn{14}{|l|}{$\begin{array}{l}\text { Sub-Study } \\
\text { SAMPLES }\end{array}$} \\
\hline $\begin{array}{l}\text { Blood Samples } \\
\text { for IG }\end{array}$ & & $x$ & & $x^{6}$ & $x^{6}$ & $x^{6}$ & $x^{6}$ & $x$ & $x$ & $x$ & $x$ & $x$ & $x$ \\
\hline $\begin{array}{l}\text { First bag (or } \\
100 \mathrm{ml} \text { ) of PEx } \\
\text { fluid for IG }\end{array}$ & & & & $x^{8}$ & & & & & & & & & \\
\hline $\begin{array}{l}\text { Blood Samples } \\
\text { for CG }\end{array}$ & & $x$ & & & & & & & & & & & $x$ \\
\hline $\begin{array}{l}\text { AE Assessment } \\
\text { (see Section 9) }\end{array}$ & & $x$ & $x$ & $\mathrm{x}$ & $x$ & $x$ & $x$ & $x$ & $x$ & $x$ & $x$ & $x$ & $x$ \\
\hline
\end{tabular}

1- Number of week(s) post treatment (i.e. post day 31) for intervention group and post randomization (i.e. post visit 1) for control group.

4- Standard of Care (SOC)

2- Assessment at 12 weeks to determine whether a second cycle is needed. If nomonitoring every 4 weeks to one year. If yes- return to day 21 (V3) with same monitoring schedule thereafter.

3-Total immunoglobulins and B cell count are required only for the IG. B cell count is not required to determine eligibility. tests

5- Please refer to the substudy Lab Manual for details

6- A blood sample before and after each plasmapheresis

7- PEx-Plasma Exchange

8- To be obtained again if a second cycle is needed

9- AEs of special interest will be collected from consent and ARs from the first dose of IMP administration until 28 days after the last intervention, and for similar duration and time-points for participants in the control group (can be conducted by phone if participants have no clinic visits). SAEs to be collected from consent until 18-month follow-up visit for both groups. 


\begin{tabular}{|c|c|c|c|c|c|c|c|c|c|c|}
\hline \multirow[t]{3}{*}{ TIMEPOINT } & \multicolumn{10}{|c|}{$\begin{array}{l}\text { Follow-Up } \\
\text { post treatment for Intervention Group, and post randomization for Control } \\
\text { Group } \\
\text { +/- one Wk (Wk=week) for samples and visits at each time-point. }\end{array}$} \\
\hline & V13 & V14 & V15 & V16 & V17 & V18 & V19 & V20 & V21 & $\begin{array}{l}\text { V22 } \\
\text { (End of } \\
\text { Study) }\end{array}$ \\
\hline & $\begin{array}{l}\text { Wk } \\
16\end{array}$ & $\begin{array}{l}\text { Wk } \\
20\end{array}$ & $\begin{array}{l}\text { Wk } \\
24\end{array}$ & $\begin{array}{l}\text { Wk } \\
28\end{array}$ & $\begin{array}{l}\text { Wk } \\
32\end{array}$ & $\begin{array}{l}\text { Wk } \\
36\end{array}$ & $\begin{array}{l}\text { Wk } \\
40\end{array}$ & $\begin{array}{l}\text { Wk } \\
44\end{array}$ & $\begin{array}{l}\text { Wk } \\
48\end{array}$ & $\begin{array}{l}18 \\
\text { month }\end{array}$ \\
\hline Concomitant medication & $\mathbf{x}$ & $\mathbf{x}$ & $\mathbf{x}$ & $\mathrm{x}$ & $x$ & 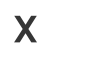 & $\mathrm{x}$ & $\mathrm{x}$ & $\mathbf{x}$ & \\
\hline Total immunoglobulins (IgG) (IG only) & & & $\mathbf{X}$ & & & $\mathrm{X}$ & & & $x$ & \\
\hline $\begin{array}{l}\text { EQ-5D, KDQoL-SF and FACT/GOG-Ntx } 4 \\
\text { questionnaires }\end{array}$ & & & & & & & & & & $\mathbf{X}$ \\
\hline \multicolumn{11}{|l|}{$\begin{array}{l}\text { Assessment of HLA Antibodies And } \\
\text { CRF }\end{array}$} \\
\hline Intervention Group (IG) & $\mathbf{x}$ & $\mathbf{x}$ & $\mathbf{x}$ & $x$ & $\mathbf{x}$ & $x$ & $x$ & $x$ & $\mathbf{x}$ & \\
\hline Control Group (CG) & & & $\mathrm{X}$ & & & $\mathbf{x}$ & & & $\mathbf{x}$ & \\
\hline \multicolumn{11}{|l|}{ Sub-Study SAMPLES } \\
\hline Blood Samples for IG & & & $\mathbf{X}$ & & & $\mathbf{X}$ & & & $\mathbf{X}$ & \\
\hline Blood Samples for CG & & & $\mathbf{X}$ & & & $\mathbf{X}$ & & & $\mathbf{X}$ & \\
\hline AE Assessment (see Section 9) ${ }^{9}$ & $x$ & $x$ & $x$ & $\mathrm{x}$ & $x$ & $x$ & $x$ & $X$ & $X$ & $X$ \\
\hline
\end{tabular}

9- AEs of special interest will be collected from consent and ARs from the first dose of IMP administration until 28 days after the last intervention, and for similar duration and time-points for participants in the control group (can be conducted by phone if participants have no clinic visits). SAEs to be collected from consent until 18-month follow-up visit for both groups.

\section{Adverse event reporting and harms $\{22\}$}

- Adverse events (AEs) of special interest (outlined in Table 5) will be collected from the time of consent until 28 days after the last intervention, and for a similar duration for participants in the control group.

- Adverse reactions (ARs) will be collected from the first dose of IMP administration and continue to be collected until 28 days after the last intervention, and for a similar duration for participants in the control group.

- Those AEs/ARs meeting the definition of a Serious Adverse Event (SAE) or Serious Adverse Reaction (SAR) will also be reported to the NHSBT CTU within 24 hours of observing or learning of the event using the trial specific electronic SAE Form. 
Table 5

Adverse events of special interest

\begin{tabular}{|c|c|c|c|c|}
\hline \multirow{2}{*}{$\begin{array}{l}\text { Adverse } \\
\text { event }\end{array}$} & \multicolumn{4}{|l|}{ Grade } \\
\hline & 1 & 2 & 3 & 4 \\
\hline $\begin{array}{l}\text { Haemoglobin } \\
(\mathrm{g} / \mathrm{L})\end{array}$ & $<$ LLN to 100 & $80-100$ & $<80$ & $\begin{array}{l}\text { Life threatening } \\
\text { consequences, } \\
\text { urgent } \\
\text { intervention } \\
\text { indicated }\end{array}$ \\
\hline $\begin{array}{l}\text { Neutrophils } \\
\left(X 10^{9} / \mathrm{L}\right)\end{array}$ & $<L L N$ to 1.5 & $1.0-1.5$ & $0.5-1.0$ & $<0.5$ \\
\hline $\begin{array}{l}\text { Platelets }(X \\
\left.10^{9} / \mathrm{L}\right)\end{array}$ & $<$ LLN to 75 & $50-75$ & $25-50$ & $<25$ \\
\hline Nausea & $\begin{array}{l}\text { Loss of appetite } \\
\text { without alteration in } \\
\text { eating habits }\end{array}$ & $\begin{array}{l}\text { Oral intake decreased } \\
\text { without significant } \\
\text { weight loss; } \\
\text { dehydration or } \\
\text { malnutrition }\end{array}$ & $\begin{array}{l}\text { Inadequate oral caloric or fluid } \\
\text { intake; iv fluids, NG feeding or } \\
\text { TPN indicated }\end{array}$ & \\
\hline Vomiting & $\begin{array}{l}\text { One or two episodes } \\
\text { (separated by } 5 \\
\text { minutes) in } 24 \text { hours }\end{array}$ & $\begin{array}{l}3-5 \text { episodes } \\
\text { (separated by } 5 \\
\text { minutes) in } 24 \text { hours }\end{array}$ & $\begin{array}{l}>6 \text { episodes (separated by } 5 \\
\text { minutes) in } 24 \text { hours; iv fluids } \\
\text { or TPN indicated }\end{array}$ & $\begin{array}{l}\text { Life threatening } \\
\text { consequences; } \\
\text { urgent } \\
\text { intervention } \\
\text { indicated }\end{array}$ \\
\hline Diarrhoea & $\begin{array}{l}\text { Increase of }<4 \text { stools } \\
\text { per day over baseline }\end{array}$ & $\begin{array}{l}\text { Increase of } 4-6 \text { stools } \\
\text { per day over baseline }\end{array}$ & $\begin{array}{l}\text { Increase of }>7 \text { stools per day } \\
\text { over baseline; incontinence; } \\
\text { limiting self-care activities of } \\
\text { daily living (ADL) }\end{array}$ & $\begin{array}{l}\text { Life-threatening } \\
\text { consequences; } \\
\text { urgent } \\
\text { intervention } \\
\text { indicated }\end{array}$ \\
\hline $\begin{array}{l}\text { Peripheral } \\
\text { motor } \\
\text { neuropathy }\end{array}$ & $\begin{array}{l}\text { Asymptomatic, clinical } \\
\text { or diagnostic } \\
\text { observations only, } \\
\text { intervention not } \\
\text { indicated }\end{array}$ & $\begin{array}{l}\text { Moderate symptoms, } \\
\text { limiting instrumental } \\
\text { ADL }\end{array}$ & $\begin{array}{l}\text { Severe symptoms, limiting } \\
\text { self-care ADL, assistive device } \\
\text { indicated }\end{array}$ & $\begin{array}{l}\text { Life threatening } \\
\text { consequences, } \\
\text { urgent } \\
\text { intervention } \\
\text { indicated }\end{array}$ \\
\hline $\begin{array}{l}\text { Peripheral } \\
\text { sensory } \\
\text { neuropathy }\end{array}$ & $\begin{array}{l}\text { Asymptomatic, loss of } \\
\text { deep tendon reflexes or } \\
\text { paraesthesia }\end{array}$ & $\begin{array}{l}\text { Moderate symptoms, } \\
\text { limiting instrumental } \\
A D L\end{array}$ & $\begin{array}{l}\text { Severe symptoms, limiting } \\
\text { self-care ADL }\end{array}$ & $\begin{array}{l}\text { Life threatening } \\
\text { consequences, } \\
\text { urgent } \\
\text { intervention } \\
\text { indicated }\end{array}$ \\
\hline Infection & $\begin{array}{l}\text { Asymptomatic or } \\
\text { requiring local } \\
\text { intervention } \\
\text { (depending on site) }\end{array}$ & $\begin{array}{l}\text { Requiring local or oral } \\
\text { treatment (depending } \\
\text { on site) }\end{array}$ & Requiring parenteral treatment & Life-threatening \\
\hline
\end{tabular}

Abbreviations: ADL, activities of daily living; iv, intravenous; LLN, lower limit normal; TPN, total parenteral nutrition

Instrumental ADLs include meal preparation, shopping, using the telephone, managing money. Self-care ADLs include bathing, dressing, using the toilet, taking medication.

\section{Events which do not require reporting as an SAE}

The following events will not be considered as SAEs and therefore will not need expedited reporting:

- Hospitalization for routine treatment or monitoring, or general care, not associated with any deterioration in condition,

- Hospitalization due to any elective surgical procedure that was planned and scheduled prior to trial entry, 
- Hospitalization, either elective or emergency, related to vascular access (excluding complications relating to vascath insertion, which will constitute a SAE),

- Transplantation,

- Events occurring at the time of dialysis and judged to be related to the dialysis.

For each SAE/SAR and Suspected Unexpected Serious Adverse Event (SUSAR) the following information will be collected:

- Full details in medical terms and case description

- Event duration (start and end dates, if applicable)

- Action taken

- Outcome

- Seriousness criteria

- Causality

- Expectedness (Expectedness assessment will only be undertaken if an event is deemed to be related)

Any change of condition or other follow-up information should be updated within the electronic SAE Form in the ITOPS database as soon as it is available or at least within 24 hours of the information becoming available. Events will be followed up until the event has resolved or a final outcome has been reached.

All SAEs assigned by the PI or delegate (or following central review) as both suspected to be related to IMP-treatment and unexpected will be classified as SUSARs and will be subject to expedited reporting to the Medicines and Healthcare Products Regulatory Agency (MHRA). The NHBT CTU will inform the Sponsor, the MHRA, and the Research Ethics Committee (REC) of SUSARs within the required expedited reporting timelines.

\section{Frequency and plans for auditing trial conduct $\{23\}$}

- The Trial Monitoring Plan has been developed and agreed by the Trial Management Group (TMG), TSC and Cl based on the trial risk assessment.

- Monitoring procedures, including regular visits to the trial sites, will be carried out by a trial monitor centrally and at sites (at least twice a year excluding initiation and close out visits) and are detailed in the trial specific monitoring plan.

- A risk assessment has been conducted which acknowledges the potential risks to the trial. This section provides an overview of the Quality Assurance (QA) and Quality Control (QC) measures that have been put in place to ensure the trial is performed and data generated and recorded in accordance with the principles of ICH GCP.

- The NHSBT CTU data managers will review all data received for errors and missing data points.

\section{Plans for communicating important protocol amendments to relevant parties (e.g. trial participants, ethical committees) $\{25\}$}

The Trial Management Group will consider protocol amendments, decide with the NHSBT CTU whether this is nonsubstantial or substantial, and communicate this to the sponsor. If the sponsor wishes to make a substantial amendment to the Clinical Trial Authorisation (CTA) or the documents that supported the original application for the CTA, the sponsor will submit a valid notice of amendment to the licensing authority (MHRA) for consideration. If the sponsor wishes to make a substantial amendment to the REC application or supporting documents, the sponsor will submit a valid notice of amendment to the REC for consideration. The MHRA and/or the REC will provide a response regarding the amendment within 35 days of receipt of the notice. Amendments will also be notified to the Health and Care Research Wales Permissions Service and communicated to the participating organisations (Research and Development office and local research team), and departments of participating sites. 


\section{Dissemination Plans $\{31$ a $\}$}

- Within one year of the end of the trial, the $\mathrm{Cl}$ will submit a final report with the results, including any publications or abstracts, to the REC. The report will include analysis and tabulation of the data.

- The final report's abstract and reference will be accessible on the NHSBT CTU website.

- Participants who indicated their interest to know the outcome will be informed by sending them a copy of the trial findings by post through their local investigators. In addition, results will be presented at national meetings and submitted for publication in specialty journals.

- Reporting will according to CONSORT recommendations. Publication of the results will be based on outcomes at 12 months following the last recruited patient. No interim publication of results is planned.

- The $\mathrm{Cl}$ and TSC will approve any publications arising from this trial, including those proposed by participating investigators.

- No data may be made public before publication and never without agreement from the $\mathrm{Cl}$ and Sponsor.

\section{Discussion}

The policy for deceased donor kidney allocation in the UK changed in September 2019. The new offering scheme was introduced to reflect the changing donor pool and to address some of the inequities observed in the previous scheme (introduced in 2006). Of particular relevance to the ITOPS study, the 2019 scheme gives preferential offering to patients with a matchability score of 10 , a cRF of $100 \%$ or an accrued waiting time of at least 7 years. If a recipient meeting these criteria (tier A) is not identified, the allocation algorithm then considers all other potential recipients (tier B). Kidneys in tier B are allocated according to a points-based system, including matchability. As a consequence, patients previously considered for enrolment in ITOPS are now much more likely to receive an offer through the national scheme compared to the 2006 scheme in place when the trial was conceived and opened to recruitment.

The DMC met in March to consider the impact of both the Covid 19 pandemic on the safety of continuing recruitment and the new allocation scheme. They recommended that recruitment be halted, but data collection from existing participants continue. The TSC reviewed and agreed to proceed with the recommendation.

\section{Trial Status}

The trial opened on $24 / 9 / 18$ and the first patient was recruited on $7 / 12 / 18$. Recruitment was halted on the recommendation of the DMC on $20 / 3 / 20$.

\section{Abbreviations}




\begin{tabular}{|c|c|}
\hline ADL & Activities of Daily Living \\
\hline AEs & Adverse events \\
\hline ARs & Adverse reactions \\
\hline CG & Control Group \\
\hline $\mathrm{cRF}$ & Calculated Reaction Frequency \\
\hline CTA & Clinical Trials Authorisation \\
\hline CTU & Clinical Trials Unit \\
\hline DMC & Data Monitoring Committee \\
\hline DSA & Donor Specific Antibody \\
\hline cRF & Calculated Reaction Frequency \\
\hline eCRF & electronic Case Record Form \\
\hline EQ-5D & European Quality of Life-5 Dimensions \\
\hline FACT/GOG-Ntx & Functional Assessment of Cancer Therapy/Gynecologic Oncology Group/Neurotoxicity \\
\hline FBC & Full Blood Count \\
\hline ICH GCP & International Conference on Harmonization Good Clinical Practice \\
\hline $\mathrm{H} \& \mathrm{l}$ & Histocompatibility and Immunogenetics \\
\hline HBV & Hepatitis B virus \\
\hline $\mathrm{HCV}$ & Hepatitis C virus \\
\hline HIV & Human Immunodeficiency Virus \\
\hline HLA & Human Leucocyte Antigen \\
\hline HR & Hazard Ratio \\
\hline HS & Highly Sensitized \\
\hline HSP & Highly Sensitized Patient \\
\hline $\lg G$ & Immunoglobulin G \\
\hline IG & Intervention Group \\
\hline IMP & Investigational Medicinal Product \\
\hline ISF & Investigator Site File \\
\hline ISRCTN & International standard randomised controlled trial number \\
\hline IVlg & Intravenous Immunoglobulin \\
\hline KDQOL-SF & Kidney Disease Quality of Life Short Form \\
\hline LFTs & Liver Function Tests \\
\hline MHRA & Medicines and Healthcare Products Agency \\
\hline MFI & Mean Fluorescence Intensity \\
\hline NHS & National Health Service \\
\hline
\end{tabular}

Page 19/24 


\begin{tabular}{|ll|}
\hline ADL & Activities of Daily Living \\
\hline NHSBT & NHS Blood and Transplant \\
\hline PEX & Plasma Exchange \\
\hline PI & Principal Investigator \\
\hline QA & Quality Assurance \\
\hline QC & Quality Control \\
\hline REC & Research and Ethics Committee \\
\hline SAE & Serious Adverse Event \\
\hline SAR & Serious Adverse Reaction \\
\hline SOC & Standard of Care \\
\hline SUSAR & Suspected Unexpected Serious Adverse Reaction \\
\hline TMG & Trial Management Group \\
\hline TSC & Trial Steering Committee \\
\hline UK & United Kingdom \\
\hline UKLKSS & UK Living Kidney Sharing Scheme \\
\hline UKTR & UK Transplant Registry \\
\hline VZV & Varicella Zoster Virus \\
\hline
\end{tabular}

\section{Declarations}

\section{Acknowledgements}

All patients who participated in the study.

Data manager: Rupa Sharma

Alison Deary, Head of Operations, NHSBT

Additional statistical advice: Matthew Robb, Cara Hudson, Dianne Bautista

Additional H\&I advice: Frances Edwards (Cardiff), Amy De'Ath (Cardiff), Surinder Day (Bristol)

Lead research nurses: Maria Coleman (Cardiff), Amanda Scott (Bristol), Sadie Smith (Leeds)

Sponsor guidance: Maureen Edgar (Cardiff)

Pharmacist advice: Dr Kathryn Murray (Cardiff)

\section{Authors' contributions \{31b\}}

SVG, RR, SD, TR, BC, DC and HW contributed to the trial design. SVG, RR, SD, TR and HW contributed to the funding acquisition. SVG, RR, SD, TR, LP, HW and SS contributed to the trial steering. SVG, RR, SD, TR, BC, and SS contributed to the data collection. SVG, RR, DI, SD, TR, SS and LP contributed to the drafting of the manuscript. SVG, RR, SD, TR, SS and LP contributed to the critical review of the manuscript. All authors read and approved the final manuscript. 


\section{Funding \{4\}}

The ITOPS study is funded by a grant from the Garfield Weston Foundation to Kidney Research UK.

\section{Availability of data and materials $\{29\}$}

Custody of the final data set will reside with the Cl and NHSBT CTU. Access to the final data set for analyses will be permitted by agreement of the TSC.

\section{Ethics approval and consent to participate $\{24\}$}

Ethical approval for the study was provided by the Wales REC 1, reference number 17/WA/0313. Written, informed consent to participate will be obtained from all participants.

\section{Consent for publication $\{32\}$}

Informed consent forms are available from the corresponding author on request.

\section{Competing interests $\{28\}$}

The Chief Investigator, Principle Investigators and all members of the trial committees declare they have no competing interests that might influence trial design, conduct or reporting.

\section{Authors' information (optional)}

SVG. Consultant Nephrologist, Cardiff and Vale University Health Board (Chief Investigator)

TR. Consultant Clinical Scientist. Head of the Welsh Transplantation and Immunogenetics Laboratory

SS. Clinical Trial Manager, NHSBT

HW. Patient advisor

LP. Senior Statistician, NHSBT

BC. Consultant Clinical Scientist, Leeds Teaching Hospital NHS Trust

DC. Medical Statistician. Associate Director Statistics and Clinical Studies, NHSBT

ME. Research Governance Coordinator, Cardiff and Vale University Health Board

KM. Clinical Trials Pharmacist

CD. Clinical Operations Manager, NHSBT

SD. Consultant Nephrologist, Leeds Teaching Hospital NHS Trust

RR. Consultant Nephrologist, Southmead Hospital, Bristol

\section{References}

1. Montgomery RL, B.E.; King, K.E.; Kraus E.S.; Kucirka, L.M.; Locke, J.E.; Warren, D.S.; Simpkins, C.E.; Daghal, N.N.; Singer, A.L.; Zachary, A.A.; Segev, D.L. Desensitisation in HLA incompatible kidney recipients and survival. New England Journal Medicine. 2011;365:9.

2. Higgins R, Lowe D, Daga S, Hathaway M, Williams C, Lam FT, et al. Pregnancy-induced HLA antibodies respond more vigorously after renal transplantation than antibodies induced by prior transplantation. Hum Immunol. 2015;76(8):54652.

3. Patel RT, P.I. Significance of the positive crossmatch test in kidney transplantation. N Engl J Med. 1969;280(14):735-9.

4. Pollinger HS, Stegall MD, Gloor JM, Moore SB, Degoey SR, Ploeger NA, et al. Kidney transplantation in patients with antibodies against donor HLA class II. Am J Transplant. 2007;7(4):857-63. 
5. Lefaucheur C, Loupy A, Hill GS, Andrade J, Nochy D, Antoine C, et al. Preexisting donor-specific HLA antibodies predict outcome in kidney transplantation. J Am Soc Nephrol. 2010;21(8):1398-406.

6. Willicombe M, Brookes P, Santos-Nunez E, Galliford J, Ballow A, McLean A, et al. Outcome of patients with preformed donor-specific antibodies following alemtuzumab induction and tacrolimus monotherapy. Am J Transplant. 2011;11(3):470-7.

7. Lawrence C, Willicombe M, Brookes PA, Santos-Nunez E, Bajaj R, Cook T, et al. Preformed complement-activating lowlevel donor-specific antibody predicts early antibody-mediated rejection in renal allografts. Transplantation. 2013;95(2):341-6.

8. Loupy A, Lefaucheur C, Vernerey D, Prugger C, Duong van Huyen JP, Mooney N, et al. Complement-binding anti-HLA antibodies and kidney-allograft survival. N Engl J Med. 2013;369(13):1215-26.

9. Manook M KL, Ahmed Z, Robb M, Johnson R, Shaw O, Kessaris N, Dorling A, Mamode, N Post-listing survival for highly sensitised patients on the UK kidney transplant waiting list: a matched cohort anaylsis. Lancet. 2017;389(10070):727734

10. Stegall MD, Gloor J, Winters JL, Moore SB, Degoey S. A comparison of plasmapheresis versus high-dose IVIG desensitization in renal allograft recipients with high levels of donor specific alloantibody. Am J Transplant. 2006;6(2):346-51.

11. Jordan SC, Vo AA, Peng A, Toyoda M, Tyan D. Intravenous gammaglobulin (IVIG): a novel approach to improve transplant rates and outcomes in highly HLA-sensitized patients. Am J Transplant. 2006;6(3):459-66.

12. Vo AA, Wechsler EA, Wang J, Peng A, Toyoda M, Lukovsky M, et al. Analysis of subcutaneous (SQ) alemtuzumab induction therapy in highly sensitized patients desensitized with IVIG and rituximab. Am J Transplant. 2008;8(1):144-9.

13. Vo A, Lukovsky M, Toyoda M, Wang J, Reinsmoen N, Lai CH, et al. Rituximab and intravenous immunoglobulin for desensitisation during renal transplantation

New England Journal Medicine. 2008;359(3):242-251

14. Vo AA, Petrozzino J, Yeung K, Sinha A, Kahwaji J, Peng A, et al. Efficacy, outcomes, and cost-effectiveness of desensitization using IVIG and rituximab. Transplantation. 2013;95(6):852-8.

15. Ejaz NS, Shields AR, Alloway RR, Sadaka B, Girnita AL, Mogilishetty G, et al. Randomized controlled pilot study of B celltargeted induction therapy in HLA sensitized kidney transplant recipients. Am J Transplant. 2013;13(12):3142-54.

16. Cicora F, Paz M, Mos F, Roberti J. Use of bortezomib to treat anti-HLA antibodies in renal transplant patients: a single centre experience. Transpl Immunol. 2013;29:71-10.

17. Aubert O, Suberbielle C, Gauthe R, Francois H, Obada EN, Durrbach A. Effect of a proteasome inhibitor plus steroids on HLA antibodies in sensitized patients awaiting a renal transplant. Transplantation. 2014;97(9):946-52.

18. Woodle ES, Shields AR, Ejaz NS, Sadaka B, Girnita A, Walsh RC, et al. Prospective iterative trial of proteasome inhibitorbased desensitization. Am J Transplant. 2015;15(1):101-18.

19. Jeong JC, Jambaldorj E, Kwon HY, Kim MG, Im HJ, Jeon HJ, et al. Desensitization Using Bortezomib and High-dose Immunoglobulin Increases Rate of Deceased Donor Kidney Transplantation. Medicine (Baltimore). 2016;95(5):1-10.

20. Tremblay S, Driscoll JJ, Rike-Shields A, Hildeman DA, Alloway RR, Girnita AL, et al. A prospective, iterative, adaptive trial of carfilzomib-based desensitization. Am J Transplant. 2020;20(2):411-421.

21. Vo AA, Choi J, Cisneros K, Reinsmoen N, Haas M, Ge S, et al. Benefits of rituximab combined with intravenous immunoglobulin for desensitization in kidney transplant recipients. Transplantation. 2014;98(3):312-9.

22. Alachkar N, Lonze BE, Zachary AA, Holechek MJ, Schillinger K, Cameron AM, et al. Infusion of high-dose intravenous immunoglobulin fails to lower the strength of human leukocyte antigen antibodies in highly sensitized patients. Transplantation. 2012;94(2):165-71. 
23. Marfo K, Ling M, Bao Y, Calder B, Ye B, Hayde N, et al. Lack of effect in desensitization with intravenous immunoglobulin and rituximab in highly sensitized patients. Transplantation. 2012;94(4):345-51.

24. Lobashevsky AL, Higgins NG, Rosner KM, Mujtaba MA, Goggins WC, Taber TE. Analysis of anti-HLA antibodies in sensitized kidney transplant candidates subjected to desensitization with intravenous immunoglobulin and rituximab. Transplantation. 2013;96(2):182-90.

25. Macklin PS, Morris PJ, Knight SR. A systematic review of the use of rituximab for desensitization in renal transplantation. Transplantation. 2014;98(8):794-805.

26. Ramos EJ, Pollinger HS, Stegall MD, Gloor JM, Dogan A, Grande JP. The effect of desensitization protocols on human splenic B-cell populations in vivo. Am J Transplant. 2007;7(2):402-7.

27. Lemy A, Toungouz M, Abramowicz D. Bortezomib: a new player in pre- and post-transplant desensitization? Nephrol Dial Transplant. 2010;25(11):3480-9.

28. Walsh RC, Brailey P, Girnita A, Alloway RR, Shields AR, Wall GE, et al. Early and late acute antibody-mediated rejection differ immunologically and in response to proteasome inhibition. Transplantation. 2011;91(11):1218-26.

29. Walsh RC, Alloway RR, Girnita AL, Woodle ES. Proteasome inhibitor-based therapy for antibody-mediated rejection. Kidney Int. 2012;81(11):1067-74.

30. Touzot M, Couvrat-Desvergnes G, Castagnet S, Cesbron A, Renaudin K, Cantarovich D, et al. Differential modulation of donor-specific antibodies after B-cell depleting therapies to cure chronic antibody mediated rejection. Transplantation. 2015;99(1):63-8.

31. Budde K, Lehner LJ. Bortezomib-based antibody reduction therapy: the first step to "true" desensitization? Am J Transplant. 2015;15(1):10-12.

32. Yabu JM, Siebert JC, Maecker HT. Immune Profiles to Predict Response to Desensitization Therapy in Highly HLASensitized Kidney Transplant Candidates. PLoS One. 2016;11(4):1-16.

33. Rajakariar R, Jivanji N, Varagunam M, Rafiq M, Gupta A, Sheaff M, et al. High pre-transplant soluble CD30 levels are predictive of the grade of rejection. Am J Transplant. 2005;5(8):1922-5.

34. Banham G, Prezzi D, Harford S, Taylor CJ, Hamer R, Higgins R, et al. Elevated pretransplantation soluble BAFF is associated with an increased risk of acute antibody-mediated rejection. Transplantation. 2013;96(4):413-20.

35. Khovanova N, Daga S, Shaikhina T, Krishnan N, Jones J, Zehnder D, et al. Subclass analysis of donor HLA-specific IgG in antibody-incompatible renal transplantation reveals a significant association of IgG4 with rejection and graft failure. Transpl Int. 2015;28(12):1405-15.

36. Schaefer SM, Susal C, Opelz G, Dohler B, Becker LE, Klein K, et al. Pre-transplant soluble CD30 in combination with total DSA but not pre-transplant C1q-DSA predictes antibody-mediated graft loss in presensitized high-risk kidney transplant recipients. HLA. 2016;87(2):89-100.

37. Zhang Y, Briggs D, Lowe D, Mitchell D, Daga S, Krishnan N, et al. A new data-driven model for post-transplant antibody dynamics in high risk kidney transplantation. Math Biosci. 2017;284:3-11.

\section{Figures}


Pre-screening

Potential participants will have completed all standard assessments and be active on the renal transplant waiting list

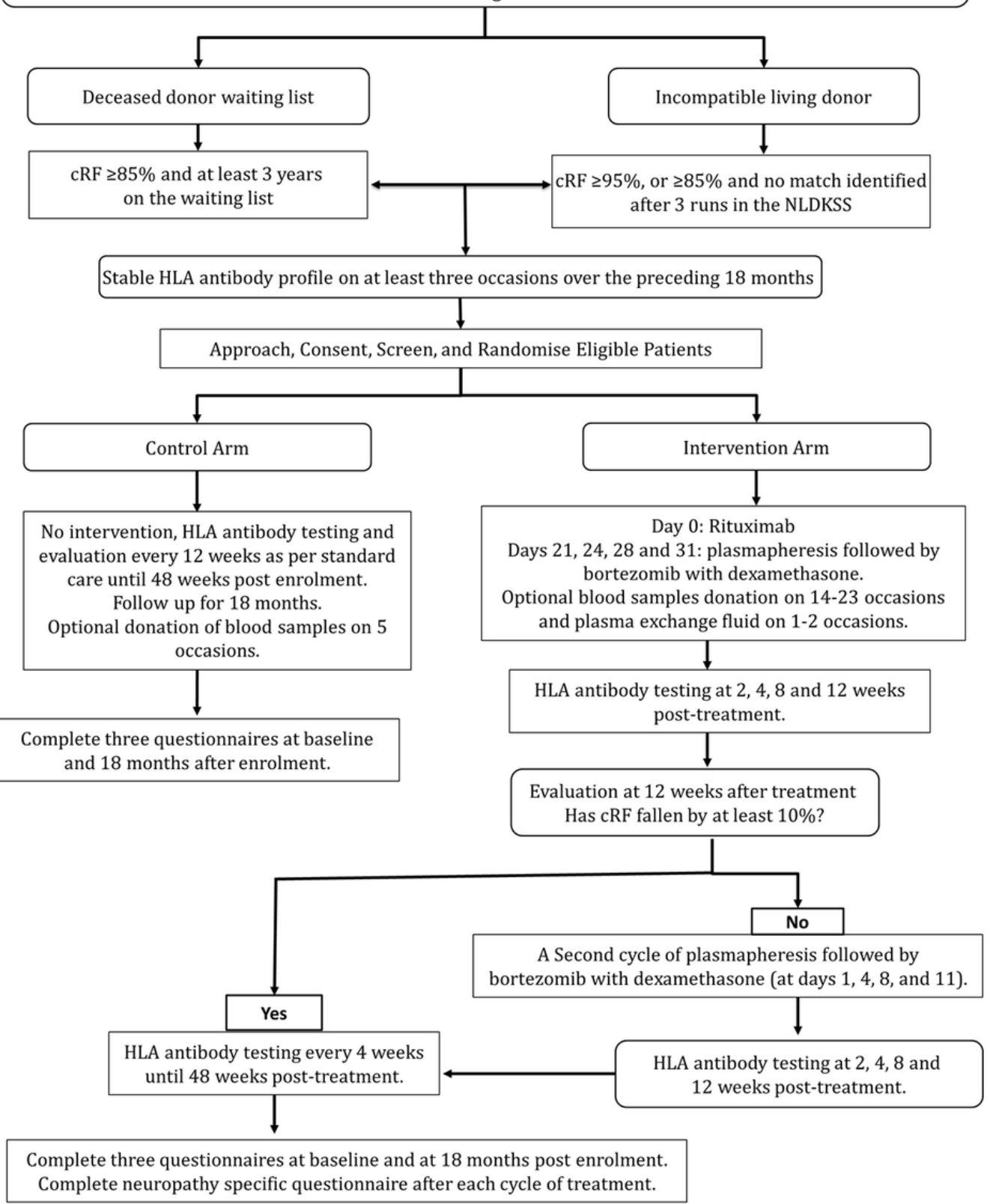

\section{Figure 1}

Participant timeline. 